\title{
ASSISTÊNCIA FARMACÊUTICA A FRENTE AOS RISCOS DO CONSUMO ABUSIVO DE REMÉDIOS PARA EMAGRECER
}

\author{
Luan Abreu de Carvalho ${ }^{1}$ \\ Leonardo Guimarães de Andrade ${ }^{2}$
}

RESUMO: A obesidade é um sério problema de saúde disseminada pelo mundo inteiro que afeta milhares de pessoas causando o aparecimento de outras enfermidades graves como hipertensão arterial, diabetes mellitus, entre outras, além de um alto número de depressão e autoestima baixa. Esses fatores associados a busca pelo corpo perfeito, acabam contribuindo para que as pessoas recorram a alternativas rápidas para a perda de peso, além disso, dentre essas alternativas, o uso de medicamentos para emagrecer (anorexígenos) não levando em consideração os riscos que tais medicamentos podem causar a saúde. Dessa forma, busca-se com o presente estudo analisar o papel do farmacêutico diante dos riscos do uso abusivo dos remédios para emagrecer.

Palavras-chave: Obesidade. Medicamentos Anorexígenos. Assistência farmacêutica.

ABSTRACT: Obesity is a serious health problem spread all over the world that affects thousands of people causing the appearance of other serious illnesses such as high blood pressure, diabetes mellitus, among others, in addition to a high number of depression and low self-esteem. These factors associated with the search for the perfect body, end up contributing to people resorting to quick alternatives for weight loss, in addition, among these alternatives, the use of drugs to lose weight (anorectics) not taking into account the risks of such drugs can cause health. Thus, this study seeks to analyze the role of the pharmacist in the face of the risks of abusive use of weight-loss drugs.

Keywords: Obesity. Anorectic drugs. Pharmaceutical care.

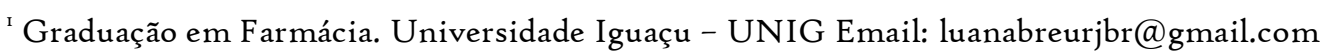
${ }^{2}$ Orientador
} 


\section{OBJETIVO GERAL}

O presente estudo tem por objetivo geral, analisar o papel do farmacêutico diante dos riscos do uso abusivo dos remédios para emagrecer.

Visa-se demonstrar a importância desse profissional para orientar a população acerca dos possíveis efeitos colaterais desses medicamentos, assim como seu uso correto.

\section{I.I OBJETIVOS ESPECÍFICOS:}

$\checkmark$ Realizar uma revisão da literatura sobre os fármacos utilizados no tratamento da obesidade;

$\checkmark$ Analisar os principais efeitos adversos dos fármacos anorexígenos;

$\checkmark$ Discorrer sobre o papel do farmacêutico na terapia farmacológica contra obesidade.

\section{I.METODOLOGIA}

A metodologia empregada neste trabalho foi a de pesquisa bibliográfica. Realizouse uma pesquisa sobre a assistência farmacêutica frente aos riscos do consumo abusivo de remédios para emagrecer.

A pesquisa foi realizada através do estudo de artigos científicos e livros virtuais anexados ao Google acadêmico, além de plataformas de bases de dados como Scientific Electronic Library Online (SCIELO), Biblioteca Virtual em Saúde (BVS), PubMed, através dos descritores: Obesidade, Medicamentos Anorexígenos, Assistência farmacêutica.

Como critérios de inclusão foram selecionados os artigos e livros da língua vernácula que fossem pertinentes ao tema e estivessem disponibilizados integralmente, e como critérios de exclusão artigos que não possuíam informações e dados relevantes para a pesquisa e aqueles que se encontravam incompletos, no período entre 2015 à 2021.

\section{JUSTIFICATIVA}

A busca por métodos rápidos e práticos para a perda de peso tem levado muitas pessoas a buscar medicamentos anorexígenos como primeira escolha de tratamento. 
No entanto, grande parte das pessoas que utilizam esses medicamentos, acabam fazendo por conta própria, sem acompanhamento adequado e sem que seja levando em consideração os riscos do uso abusivo desses fármacos.

Nesse sentido, o presente estudo se justifica uma vez que, busca alertar como o uso indiscriminado de anorexígenos pode ocasionar sérios danos à saúde, tanto em termos físicos quanto emocionais.

Dada a relevância do tema, espera-se contribuir para a ampliação do conhecimento destes fármacos, bem como seus efeitos, riscos e contraindicações.

\section{INTRODUÇÃO}

Um dos grandes problemas de saúde pública da atualidade está relacionado a obesidade, que se caracteriza se por acúmulo excessivo de tecido adiposo, principalmente na região abdominal. Estima-se que no mundo 650 milhões de pessoas adultas são obesas (WHO, 2018). No Brasil, os dados da Pesquisa Nacional de Saúde - 2019 apontam que uma em cada quatro pessoas de I8 anos ou mais de idade estão obesas, o equivalente a 4I milhões de pessoas, e cerca de 96 milhões de pessoas (6o,3\%) estão com excesso de peso (CAMPOS, 2020).

Além de ser considerada uma doença crônica, a obesidade também é fator de risco para outras enfermidades, como hipertensão arterial, diabetes mellitus, acidente vascular encefálico, doenças cardiovasculares, neoplasias, doença hepática gordurosa não alcoólica entre outras. Juntamente com os danos causados à saúde, associa-se a obesidade o aparecimento de depressões, autoestima baixa, pouca ou nenhumas perspectivas de relacionamento social, isso se deve principalmente, ao preconceito com a pessoa que está acima do peso. (AZEVEDO \& BRITO, 2018, ANDRADE Et Al., 2019).

Nesse sentido, o auto índice de obesidade entre a população e os problemas associados a ela, bem como, a busca para atender os padrões de beleza impostos pela sociedade e a preocupação com a imagem, entre outros fatores, tem levado a população a buscar alternativas rápidas para a perda de peso, dentre essas alternativas, o uso de medicamentos para emagrecer (anorexígenos) tem sido apontado como uma das soluções imediatistas e "milagrosas" as quais as pessoas mais recorrem para alcançar esses objetivos (SILVA; OLIVEIRA; FERREIRA, 2015). 
Dentre os países consumidores de medicamentos anorexígenos, o Brasil ocupa a primeira posição. De acordo com o relatório anual da Organização das Nações Unidas (ONU) no Brasil são consumidas I2,5 doses diárias dessas substâncias, contra II,8 na Argentina, 9,8 na Coréia do Sul e 4,9 nos Estados Unidos. Entre os medicamentos mais utilizados para a perda de peso aprovados pela Agência Nacional de Vigilância Sanitária ANVISA, estão a anfepramona, o femproporex, o mazindol e a sibutramina, os quais agem sobre o sistema nervoso central liberando substâncias que transmitem a sensação de ausência de fome (ANDRADE Et Al., 2019).

No entanto, a grande problemática envolvendo a questão dos anorexígenos é que, o tratamento farmacológico só é indicado como prática adjuvante ao tratamento da obesidade, diante das falhas das terapias não farmacológicas (mudança de hábitos alimentares, prática de exercícios físicos e intervenção do profissional nutricionista) ou em decorrência de comorbidades associadas à obesidade ou em casos de obesidade tipo 2 e 3 (COSTA; DUARTE, 2017).

O que se percebe é que esses medicamentos têm sido usados de maneira abusiva e irracional por pessoas que buscam alternativas simples e rápidas para o emagrecimento, o que pode ocasionar inúmeras consequências para a saúde (SANTOS Et Al., 2019).

No caso dos remédios anorexígenos, a automedicação é perigosa, podendo levar à dependência física, causar efeitos colaterais sobre a função mental e comportamental. É de extrema relevância que os mesmos sejam utilizados com as devidas orientações médicas e terapêuticas (SILVA, OLIVEIRA, FERREIRA, 2012).

Nesse sentido, o farmacêutico tem papel importante na orientação dos riscos e benefícios dos fármacos anorexígenos, principalmente, no que diz respeito aos problemas relacionados ao uso incorreto e abusivo desses medicamentos. Através da atenção farmacêutica, esse profissional junto com a equipe multidisciplinar, pode propor o tratamento adequado de acordo com a necessidade de cada paciente, visando obter os melhores resultados para os mesmos (SILVA, MAGALINI e SANTOS, 2018). 


\section{EFEITOS ADVERSOS DO CONSUMO DE MEDICAMENTOS PARA EMAGRECER}

Os medicamentos anorexígenos são considerados coadjuvantes para o tratamento da obesidade, associados principalmente, as modificações de estilo de vida, como dieta e prática de exercícios físicos. (SILVA Et Al., 2018).

No entanto, o uso desses medicamentos nem sempre está relacionado a essa condição clínica, muitas pessoas se automedicam fazendo uso dos anorexígenos apenas para fins estéticos, sem levar em consideração os inúmeros efeitos colaterais que podem causar sérios danos à saúde (SANTANA, 2016).

Fortes Et Al. (2015) relatam que a crescente prática de automedicação e uso abusivo dos anorexígenos no Brasil, levaram a discussão de medidas para o controle e comercialização desses fármacos, que tem os efeitos colaterais e risco de dependência aumentados com a prática da automedicação.

De acordo com Silva Et Al. (2018) o uso indiscriminado desses remédios pode acarretar inúmeros efeitos colaterais como ansiedade, insônia, alterações do humor, comportamento violento, e alguns distúrbios psicóticos como paranoia e alucinações visuais.

Os estudos realizados por Conceição Et Al. (2017) apontaram para efeitos adversos relacionados ao sistema gastrointestinal no qual foram relatados a ocorrência de náuseas, vômitos e diarreia e ao sistema cardiovascular, onde foi constatado leve aumento da frequência cardíaca $(\mathrm{I}-3 \mathrm{bpm})$.

Em relação aos efeitos colaterais dos fármacos anorexígenos mais utilizados no Brasil, Moreira e Alves (2015) ressaltam que a ocorrência de dependência física e psíquica relacionados a esses medicamentos é alta, tornando seu uso alvo de muitas controvérsias, principalmente no que concerne a capacidade de se tornarem perigosos.

De acordo com os autores a sibutramina, um dos fármacos mais utilizados para emagrecer, provoca reações adversas como boca seca, aumento da pressão arterial, palpitação, anorexia, insônia, vertigens e reações de hipersensibilidade (MOREIRA E ALVES, 2015).

Ainda sobre a sibutramina, Rang e Dale (2020) relatam que entre os efeitos colaterais do uso da sibutramina estão dor de cabeça, boca seca, náusea, aumento da 
sudorese, dispneia, constipação intestinal, vertigem, alteração no paladar, aumento da frequência cardíaca, hipertensão arterial, pressão arterial sistólica e diastólica de repouso.

Lima Et Al. (2018) ressaltam que a sibutramina é contraindicada em casos de pacientes que possuem doença arterial coronariana ou periférica, acidente vascular cerebral e hipertensão arterial descontrolada, além do exposto, esse medicamento não é indicado para indivíduos que possuem doenças relacionadas ao sistema nervoso central

Andrade Et Al. (2019) ressaltam que a anfepramona provoca insônia, agitação e nervosismo, há relatos de casos onde o sujeito apresenta alucinações, delírios e psicoses, geralmente esses episódios estão relacionados a casos de intoxicação aguda.

Já Duarte Et Al. (2020) afirmam que a anfepramona causa taquicardia, dor de cabeça, náusea, boca seca, vomito, constipação intestinal, redução da libido e impotência sexual.

De acordo com Lucas (2019) para pacientes com idade inferior a 40 anos como histórico de doenças cardiovasculares, tabagismo, dislipidemias e uso de substâncias simpatomiméticas, sendo lícitas ou ilícita, devem ficar atentos e não fazerem uso desses medicamentos.

Em relação ao femproporex as reações mais comuns associadas a esse medicamento são casos de hipertensão arterial e pulmonar, glaucoma, náuseas, vômitos, aumento da ansiedade, cefaleias, excitação e palidez (CUNHA Et Al., 202I).

Nos estudos de Duarte Et Al. (2020) outros efeitos são mencionados como taquicardia, palpitação, hipertensão, boca seca, desconforto abdominal, vomito, diarreia, convulsões, episódios psicóticos, depressão, visão turva, irritabilidade, além de alterações comportamentais e cardiovasculares, alopecia e arritmia, que pode até levar ao colapso cardiovascular.

O femproporex é contraindicado para pacientes com forma grave e descontrolada de hipertensão, além daqueles com insuficiência cardíaca e histórico de doenças cardiovasculares, glaucoma e doenças psiquiátricas (LUCAS, 2019).

Estudos apontam que o mazindol pode causar alguns efeitos como constipação, nervosismo, inquietação, vertigem, taquicardia e, de forma menos frequente cefaleia, aumento da sudorese e alteração no paladar (RODRIGUES Et A1., 2010). 
Além desses efeitos, de acordo com Duarte Et Al. (2020) o mazindol pode provocar Insônia, dor de cabeça, boca seca, náusea, arrepios, irritabilidade, fraqueza, desconforto gástrico, tontura, vertigem e hiperidrose.

De acordo como o relatório da Agência Nacional de Vigilância Sanitária ANVISA, publicada pelo Ministério da Saúde, o mazindol apresenta mais riscos do que benefícios, dessa forma, seu uso a longo prazo é inviável, sendo contraindicado em casos de pacientes que já apresentam histórico de uso abusivo de medicamentos, doenças cardiovasculares, glaucoma e pacientes com histórico de estado de euforia (BRASIL, 20II).

Os efeitos adversos bem como as contraindicações devem ser observados na escolha do medicamento. Nesse sentido, seu uso abusivo e indiscriminado não leva em consideração tais efeitos, agravando ainda mais os problemas de saúde, além de não alcançar os efeitos desejáveis (CUNHA Et Al., 202I).

\section{ASSISTÊNCIA FARMACÊUTICA}

A questão da obesidade e sua relação com o consumo abusivo de remédios para emagrecer é um problema complexo, que envolve questões de saúde, fatores psicológicos como autoestima baixa, depressão, ansiedade, fatores genéticos, metabólicos e hormonais, entre outros fatores que demandam acompanhamento profissional multidisciplinar, como nutricionistas, psicólogos e farmacêuticos (MENDES, 2018).

Nesse cenário, o farmacêutico por ser o profissional mais acessível a população, tem destacado no papel do cuidado à saúde e atenção ao paciente, sendo o profissional mais capacitado acerca dos medicamentos e suas informações, contribuindo para eficácia no tratamento, minimizando os erros durante o uso desses fármacos (RADAELLI, 2016).

De acordo com Rodrigues Et Al. (2018) a atenção farmacêutica voltada para os pacientes que fazem uso desses medicamentos é fundamental, pois através da educação em saúde, o farmacêutico pode prestar a orientação sobre os benefícios e malefícios desses medicamentos. Além disso, o farmacêutico avalia o tratamento juntos com outros profissionais discutindo a melhor medicação a ser empregada de acordo como a necessidade de cada paciente (BARROS, 2019). 
De acordo com Lima Et Al. (2018) e Andrade Et Al. (2019) a atuação do farmacêutico na dispensação dos fármacos é de fundamental relevância visto que no ato dispensação de um medicamento o farmacêutico poderá informar ao paciente a forma correta e racional de fazer uso do medicamento, esclarecer todas as dúvidas com a finalidade de um resultado eficaz e seguro, afim de promover a promoção e recuperação da saúde.

Andrade Et Al. ressaltam ainda que no momento da dispensação desses fármacos, o farmacêutico pode sanar todas as dúvidas e alertar a respeito do uso indiscriminado e a superdosagem destes medicamentos. Além disso, o autor destaca a importância desse profissional como conscientizador podendo realizar campanhas visando a educação e motivação do paciente em relação ao tratamento correto e o uso racional desses fármacos.

Não menos importante, cabe ao farmacêutico conscientizar o paciente sobre as outras formas de tratamento da obesidade, apontando para a importância das mudanças de hábitos diários, com a introdução de prática de atividades físicas, bem como, esclarecendo a importância do acompanhamento de outros profissionais como nutricionista para a introdução de hábitos alimentares saudáveis, entre outros (COSTA, 2019).

\section{CONCLUSÃO}

A busca para o tratamento da obesidade envolve inúmeros aspectos e está ligada principalmente ao emagrecimento saudável, que requer um processo constante de reeducação alimentar, aliada a prática de atividades físicas, mudanças de hábitos de vidas que devem ser incorporadas no dia a dia do paciente obeso ou daqueles que desejam ter um corpo mais saudável.

O tratamento farmacológico é recomendado apenas como uma terapia auxiliar em casos em que as atividades físicas e mudanças alimentares não alcançarem os efeitos desejados ou em casos de obesidade associada a comorbidades.

Nesses casos, faz-se essencial a intervenção multidisciplinar, com o acompanhamento de profissionais como nutricionistas, psicólogos, endocrinologistas, educadores físicos e principalmente os farmacêuticos.

Esses profissionais estão capacitados para prestar as orientações relacionadas aos medicamentos anorexígenos, alertando para a necessidade de cautela no uso desses 
medicamentos, principalmente por apresentarem contraindicações e efeitos colaterais, devendo, portanto, serem utilizados apenas quando os tratamentos não-farmacológico não funcionar ou em situações onde a obesidade pode ocasionar danos irreparáveis a saúde.

Destaca-se a relevância do tema diante do auto índice de consumo abusivo desses medicamentos no país, assim espera-se contribuir para que os usuários desses medicamentos se torem conscientes dos possíveis danos que os mesmos podem causar a saúde, evitando assim, efeitos contrários aos desejados, que é acima de tudo o tratamento da obesidade e o emagrecimento de forma saudável, espera-se também, destacar a importância do acompanhamento da equipe multidisciplinar, em especial do farmacêutico, para que sejam sanadas todas as dúvidas do paciente, e por fim, espera-se a ampliação das discussões a respeito dos riscos que das medicações consideradas milagrosas pela população, podendo assim minimizá-los.

\section{REFERÊNCIAS}

ANDRADE TB, ANDRADE GB, HONORATO DE JESUS J, SILVA JN. O farmacêutico frente aos riscos do uso de inibidores de apetite: a sibutramina. Revista da Faculdade de Educação e Meio Ambiente - FAEMA, Ariquemes, v. Io, n. I, p. 81-92, jan.jun. 2019.

AZEVEDO, F. R. \& BRITO, B. C. Influência das variáveis nutricionais e da obesidade sobre a saúde e o metabolismo. Revista da Associação Médica Brasileira (English Edition). 58(6): 714-23. 2018.

BARROS, Y. I. B. Imagem corporal e a influência da mídia na construção do corpo feminino. Monografia, Universidade Católica de Salvador. 2019.

BRASIL. Ministério da Saúde. ANVISA. (20II). Avaliação de eficácia e segurança dos medicamentos inibidores do apetite. Disponível em: http://portal.anvisa.gov.br/documents21920I/2782895/Nota+t\%C3\%A9cnica+anorex\% $\mathrm{C}_{3}$ $\% A D g e n o s / 16367 d 39 f 947-40 f e-a 89 f-65 a 51 b 465792$ Acesso em 21 set. 2021.

CAMPOS, A, C. IBGE: obesidade mais do que dobra na população com mais de 20 anos. Resultados da Pesquisa Nacional de Saúde 2019. Agência Brasil, 2020. Disponivel em: https:agenciabrasil.ebc.com.br/saude/noticia/ibge-obesidade-mais-do-que - dobra-napopulacao-com-mais-de-20-anos. Acesso em I5.set.2021.

CONCEIÇÃO, R. A. DA; SILVA, P. N. DA; BARBOSA, M. L. C. Drugs for the Treatment of Type II Diabetes: A Visit to the Past and a Look to the Future. Revista Virtual de Química, v. 9, n. 2, p. 514-534, 2017. 
COSTA, A. M. J.; DUARTE, S. F. P. Principais Medicamentos Utilizados no tratamento da Obesidade e Vias de Ação: Uma Revisão Sistemática. Id on Line Revista de Psicologia, v. II, n. 35, p. 199-209, 2017

COSTA, J.C. O uso da sibutramina no tratamento da obesidade: uma revisão literária / Monografia. Faculdade Maria Milza. Governador Mangabeira - BA, 2020

CUNHA, T.M.M.; SESTITO, V.M.B.; CAMPANHA, I.O.; MOREIRA, K.L.; PEREIRA, G.J.V. Riscos e efeitos colaterais do uso de anorexígenos em mulheres no estado de São Paulo. Research, Society and Development, v. Io, n. 13, 2021.

DUARTE, A. P. N. B.; GOVATO, T. C. P.; CARVAlHO, R. G.; PONTES-JÚNIOR, L. C. B.; RODRIGUES, C. L.; SANTOS, G. M. P.; NICOLAU, L. A. D.; FERRAZ, R. R. N. \& MENEZES-RODRIGUES, F. S. Uso de anfepramona, femproporex, mazindol e sibutramina no tratamento de pacientes com sobrepeso ou obesidade: análise farmacológica e clínica. International Journal of Health Management Review, v.8 n. 6. 2020.

FORTES R.C, GUIMARÃES NG, HAACK A. Orlistat e sibutramina: bons coadjuvantes para perda e manutenção de peso? Rev Bras Nutr Clin 2015.

LIMA, T. A. M.; FRANCO, T. F.; PEREIRA, L. L. V.; GODOY, M. F. Análise das prescrições de sibutramina em drogaria. Revista Eletrônica de Farmácia, v. I5, 2018.

LUCAS, B.B. Farmacoterapia da obesidade: uma revisão da literatura. /

Bárbara Belmiro Lucas. - Cuité: CES, 2019.

MENDES, C. F. O. Assistência farmacêutica na obesidade: uma nova análise. Monografia, $87 \mathrm{f}$. Universidade Federal de Ouro Preto. 2018

OLIVEIRA KR, VILELA PA, FREITAS JGA, SANTOS UG. Sibutramina: efeitos e riscos do uso indiscriminado em obesos. Rev. Eletr. Trab. Acad.: Universo. 2016; I(3): 291302

RADAELLI M, PEDROSO RC, MEDEIROS LF. Farmacoterapia da obesidade: benefícios e riscos. saúde e desenvolvimento humano. 20I6; 4(I): IOI-II5.

RANG, H.P; DALE, M.M. Farmacologia. 9ª edição. GEN Guanabara Koogan, 2020.

SANTANA, C; WANDERLEY, H C. O uso farmacológico de anfepramona e Sibutramina no tratamento coadjuvante da obesidade. Congresso Brasileiro de Ciências da Saúde. Editora realize. Revistas conbracis, 2016.

SANTOS, KP, SILVA GE, MODESTO KR. Perigo dos medicamentos para emagrecer. Rev Iniciação Cientifica e Extensão. 2019; 2(I): 37-45. 
SILVA, A. C.; MAGALINI, A. P.; SANTOS, D. C. F. Efeitos da sibutramina sobre o sistema reprodutor feminino de ratas Wistar. Revista Brasileira Multidisciplinar, v. 2I, n. I, p. 95-102, 2018.

SILVA, G.A.; XIMENES, R.C.C.; PINTO, T.C.C et al. Consumo de formulações emagrecedoras e risco de transtornos alimentares em universitários de cursos de saúde. $\mathrm{J}$ Bras Psiquiatr. 2018;68(4):239-46

SILVA, J. R.; OLIVEIRA, E. N. F.; FERREIRA, A. G. Avaliação do consumo de anorexígenos derivados de anfetamina em cidades de Goiás-GO. Ensaios e Ciência: C. Biológicas, Agrárias e da Saúde, v. ı6, n. 3, 2012.

SILVA, R.N. SANTANA, C. A.; MARTINS, T.S. Uso Dos Anorexígenos, Seus Riscos E Farmacologia Para O Tratamento Da Obesidade. Revista Científica Multidisciplinar Núcleo do Conhecimento. Ano o6, Ed. 04, Vol. or, pp. I24-I40

WHO. (2018). Obesity and overweight [internet]. Genebra: World Health Organization (WHO). Disponivel em http://www.who.int/newsroom/factsheets/detail/obesity-andoverweight Acesso em I5.set.2021. 